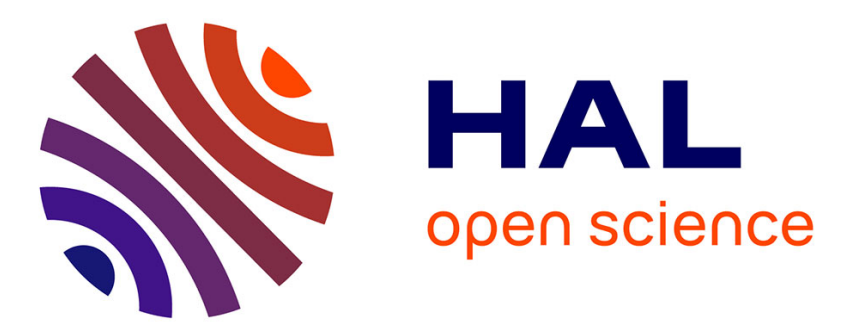

\title{
Do parental reports of child hyperkinetic disorder symptoms at school predict teacher ratings?
}

\author{
Kapil Sayal, Robert Goodman
}

\section{To cite this version:}

Kapil Sayal, Robert Goodman. Do parental reports of child hyperkinetic disorder symptoms at school predict teacher ratings?. European Child and Adolescent Psychiatry, 2009, 18 (6), pp.336344. 10.1007/s00787-009-0735-y . hal-00478094

\section{HAL Id: hal-00478094 https://hal.science/hal-00478094}

Submitted on 30 Apr 2010

HAL is a multi-disciplinary open access archive for the deposit and dissemination of scientific research documents, whether they are published or not. The documents may come from teaching and research institutions in France or abroad, or from public or private research centers.
L'archive ouverte pluridisciplinaire HAL, est destinée au dépôt et à la diffusion de documents scientifiques de niveau recherche, publiés ou non, émanant des établissements d'enseignement et de recherche français ou étrangers, des laboratoires publics ou privés. 


\section{Kapil Sayal Robert Goodman}

\section{Do parental reports of child hyperkinetic disorder symptoms at school predict teacher ratings?}

Received: 20 May 2008

Accepted: 12 October 2008

Published online: 6 February 2009
K. Sayal ( $\square)$

Section of Developmental Psychiatry,

E Floor, South Block,

Queen's Medical Centre

University of Nottingham

Nottingham NG7 2UH, UK

E-Mail: kapil.sayal@nottingham.ac.uk

R. Goodman

Department of Child Psychiatry,

Institute of Psychiatry

King's College London

Denmark Hill, London
Abstract Background Clinical practice guidelines for the evaluation of children with suspected hyperkinetic disorder or ADHD recommend that information is collected from teachers. Methods Using the development and well-being assessment, parents of 5-16 year olds participating in the 1999 and 2004 British Child and Adolescent Mental Health Surveys were asked about symptoms relating to hyperkinetic disorder and reports of teacher complaints about these symptoms. We examined whether parental reports about symptoms at school reflect teacher ratings and can be relied upon by clinicians. Results Parent reports about symptoms at school were moderately correlated with teacher ratings. If children potentially met criteria for hyperkinetic disorder based on parental ratings only, the positive predictive value (PPV) for a research diagnosis of hyperkinetic disorder was $47 \%$. When parents reported high levels of symptoms at school in addition to sufficient parent-rated symptoms and impairment, the PPV for a diagnosis of hyperkinetic disorder increased to $59 \%$. Conclusions In a community sample, we found that parental reports about symptoms at school have limited utility in predicting teacher ratings. Our findings highlight that it is desirable and worthwhile for clinicians to obtain direct information from the teacher. If this is unavailable, clinicians and researchers should be aware that "second-hand" information about symptoms at school is second best.

Key words hyperkinetic disorder - ADHD - symptoms parent - teacher

\section{Introduction}

For children with hyperkinetic disorder (HKD) or attention deficit/hyperactivity disorder (ADHD), accurate clinical diagnosis is an essential prerequisite to receiving evidence-based treatments. As parental concerns and perceptions of problems play a major role in determining presentations to services $[16,18]$, these might influence how clinicians interpret parental accounts of symptoms including indirect reports about possible difficulties at school. However, this may be at the expense of accurate clinical identification. Misdiagnosis carries risks such as commencing potentially long-term treatment unnecessarily and the inappropriate labeling of these children. There have been concerns about the levels of inappropriate medication use in countries such as the United States, Canada, and Australia [14, 20]. This raises the question of whether parental report about symptoms at 
school can give an accurate indication of teacher ratings and assist diagnostically.

In European practice, the diagnostic category of HKD has been used traditionally [21, 25]. This approximates to a severe form of the combined subtype of ADHD. Although there is increasing public and professional use of the term ADHD, children who also meet criteria for HKD are most likely to receive treatment in practice $[11,19]$. Compared to children with ADHD, children who also meet criteria for HKD have more severe symptoms, are more impaired in terms of academic and cognitive functioning, and have a greater response to medication treatment [15, $22]$. As the diagnostic criteria specify the presence of hyperactivity, impulsivity and inattention symptoms that are pervasive across situations, it is desirable that information is collected about the child's symptoms across different settings. Clinical practice guidelines recommend that the evaluation of children with suspected ADHD/HKD (the broader group of children possibly meeting criteria for either diagnosis) includes contact with teachers $[1,21]$. However, this might be difficult in practice in situations where the teacher is new, the child has several teachers, the teacher does not complete the requested information or is unavailable (e.g. during school holidays), or the parent does not want the school to be contacted or to know about the clinical assessment. Although it is highly desirable to obtain teacher ratings whenever possible, we investigate the utility of proxy parent reports when teacher ratings are absent.

Although routine enquiries of parental reports about their child's symptoms at school can readily be made in clinics, it is uncertain whether clinicians can rely on these parental reports when making a diagnosis. If it could be demonstrated that parents are reliable informants about symptoms at school, these accounts could be useful for clinicians and researchers and perhaps suffice if teacher information is unavailable. Recent studies that have compared parent reports about ADHD symptoms at school with teacher ratings have found low to moderate levels of correlation between these two sets of ratings with parents under-estimating the severity of symptoms at school $[5,13,17]$. However, these studies have involved referred samples or children selected on the basis of pervasiveness of symptoms which may limit the generalisability of their findings. This investigation of the predictive utility of parent reports about HKD symptoms at school matches the way in which clinicians might use these accounts in practice. The issue of whether parents provide a useful account about their child's symptoms at school is most salient for those children whose parents report symptoms at home. In a large, representative, epidemiological community sample, we investigate: (1) how well parent reports about symptoms at school predict teacher ratings, (2) whether child factors (gender and age) influence this relationship, and (3) whether parent reports about symptoms at school assist diagnostically.

\section{Methods}

\section{Sample and procedure}

We used pooled data from the two British Child and Adolescent Mental Health Surveys carried out in 1999 and 2004 which aimed to provide prevalence estimates to inform national service planning $[7,11,12]$. These surveys involve a nationally representative sample of 5-16 year olds identified through child benefit records. Data were obtained on 83 and $76 \%$ of the target sample in each survey respectively $[11,12]$, resulting in a final sample of 18,415 children.

As part of the structured interview administered by trained interviewers (see "Measures"), parents of all 18,415 children completed two sets of screening questions about HKD. First, they were asked whether their child definitely has problems with overactivity or poor concentration. Second, they also completed the strengths and difficulties questionnaire (SDQ) which is a well-validated measure of childhood mental health $[8,9]$. It includes a hyperactivity/inattention scale (score range 0-10) with five items (each scored 0-2): "restless, overactive, cannot stay still for long"; "constantly fidgeting or squirming"; "easily distracted, concentration wanders"; "thinks things out before acting"; and "sees tasks through to the end, good attention span". In line with clinical practice guidelines when considering the possibility of HKD in a general mental health assessment, a skip rule was created whereby only parents endorsing the first screening question or providing a SDQ score of at least 6 were asked more detailed questions about hyperkinetic disorder. Hence, the screening to identify the sub-sample for more detailed enquiry was based on six questions. These cut-offs were established using pilot study data gathered on both referred and community cases who were interviewed without any skip rules [12]. The survey methodology aimed to maximise the number of true positive cases at the expense of increasing the number of true negatives about whom more detailed questions were asked. Overall, 4,814 (26\%) children were identified by this skip rule. Of these, $3126(65 \%)$ were male and their mean age was 10.02 years (standard deviation $=3.22$ ).

Most (96\%) participating families gave permission to contact teachers. Most teachers were only asked to complete ratings on one child-figures from the 1999 
survey indicated that there was only one participating child in half of the schools approached [6]. In terms of timing, all teacher data were collected between January and May to avoid the start of the academic year (September) when teachers do not know pupils as well. Parent data were collected over the same period.

\section{Measures}

The research diagnosis of $\mathrm{HKD}$ was based on the reliable and well-validated development and wellbeing assessment (DAWBA) which is a structured combined package of parental interview and teacher questionnaire [10]. The questions are closely related to ICD-10 and focus on current problems [25]. Verbatim accounts of reported problems were also elicited from parents. Experienced clinicians reviewed all the available information in assigning a DAWBA diagnosis of HKD based on ICD-10 criteria. This approach emulated the clinical diagnostic process as closely as possible [7]. The prevalence of a DAWBA diagnosis of HKD was $5 \%$ amongst the 4,814 children identified by the skip rule and $5.2 \%$ amongst the 3,648 children who also had teacher data ( $76 \%$ teacher response rate). Using the HKD section of the DAWBA, the following measures were collected from the parents and teachers of children identified by the skip rule:

1. Parent and teacher ratings of each of the 18 ICD-10 symptoms $[10,25]$. These are described as "ratings" in the paper. For the analyses, a parent-rated symptom was coded as being present if they endorsed it as occurring "a lot more" over the last 6 months than other children of the same age. A teacher-rated symptom was coded as being present if they endorsed it as being "certainly true". The number of symptoms by domain were: inattention (0-9), hyperactivity (0-5), and impulsivity (0-4).

2. Impairment. Parents were asked four items relating to home life, friendships, classroom learning, and leisure activities. The teacher was asked about friendships and classroom learning. These items were rated on a 4-point scale: "not at all," "a little," "a medium amount," and "a great deal."

3. Parents reports about symptoms at school (these are described as "parent reports" in the paper). Parents were asked to report if the teacher had complained of any of the following problems in the last 6 months: (a) poor concentration or being easily distracted (inattention), (b) fidgetiness, restlessness, or overactivity (hyperactivity), and (c) acting without thinking, frequently butting in, or not waiting for their turn (impulsivity). These domains were each rated as "no", "a little" or "a lot" (scored as $0-2$ ), giving a total score of 0-6.

\section{Data analysis}

1. The correlations were examined between the score (0-2) for parent reports about each domain (inattention, hyperactivity, impulsivity) of symptoms at school and the total number of parent- and teacher-rated symptoms. To enable comparison of findings across the three sets of correlation coefficients, we treated the parent report about symptoms at school scale as a continuous variable and used Pearson's correlation coefficients. This provides an indication of the inter-relationships between parent reports of symptoms and parent and teacher ratings. These analyses were repeated by child age (ages 5-10 and 11-16 years, reflecting transitions in the educational system in the UK) and gender to examine whether these factors influence the prediction.

2. The positive predictive value (PPV) was calculated to show the probability that teacher ratings for the presence of a sufficient number of symptoms for each domain of HKD will be positive given that the parent report about symptoms at school is positive ("a lot"). The PPV is appropriate for samples where all children score above the cut-off for a particular informant [17] and is a useful clinical guide as to how predictive an affirmative parent report is about the presence of symptoms at school. Conversely, negative predictive values (NPV) were calculated to show the probability that the teacher does not rate a sufficient number of symptoms for each domain of HKD, given that the parent report about symptoms at school is negative ("no" or "a little"). The approach to calculating PPVs and NPVs is shown in Table 1. The diagnostic criteria for HKD specify that symptoms should be pervasive but do not specify how many symptoms need to be present in each situation. This could be interpreted, at one extreme, as

Table 1 Calculations of positive and negative predictive values

\begin{tabular}{lll}
\hline & Teacher ratings of sufficient symptoms (yes) & Teacher ratings of sufficient symptoms (no) \\
\hline Parent report about symptoms at school ("a lot") & $a$ & $b$ \\
Parent report about symptoms at school ("no" or "a little") & $c$ & $d$ \\
\hline
\end{tabular}

Positive predictive value (PPV $=a / a+b)$; negative predictive value $(\mathrm{NPV}=d / c+d)$ 
requiring the full symptom criteria (involving a minimum of six inattention, three hyperactivity, and one impulsivity symptoms) to be met according to both parent and teacher ratings to, at the other extreme, requiring just one symptom to be confirmed by the second rater. Therefore, with teacher ratings as the outcome, we examined the predictive values of parent reports about symptoms at school for the confirmation of pervasiveness in three ways: (1) a strict approach requiring the teacher to endorse the presence of at least $6+3+1$ symptoms (ICD-10 domain-specific minimum), (2) a middle approach tested in two ways with the teacher endorsing: (a) at least $3+2+1$ symptoms and (b) any six symptoms, and (3) a loose approach involving just one teacher-rated symptom.

3. We then examined how parental reports about symptoms at school might assist diagnostically. This is clinically relevant as the parental account obtained in the clinic might be highly suggestive of HKD but a report corroborating pervasiveness is still required from the teacher. Amongst the children with both parent and teacher data, we developed a parent-only diagnosis of HKD based on parent ratings of at least $6+3+1$ symptoms (domain-specific minimum) as well as at least "a medium amount" of impairment in one area. With the DAWBA diagnosis of HKD as the outcome, we examined the PPV and NPV of the parent-only diagnosis. We then investigated the effect of adding in parent reports about school using receiver operator characteristic (ROC) curves to explore the point on the total 0-6 scale at which parent reports of symptoms at school best predicted whether the child has a DAWBA diagnosis of HKD. At each point on the ROC curve, the predictive utility of parent report about symptoms at school is reported in terms of: (a) sensitivity (probability that the parent report is positive given that child has
HKD), (b) specificity (probability that the parent report is negative given that the child does not have HKD), (c) PPV (probability that the child has HKD given that the parent report is positive), (d) NPV (probability that the child does not have HKD given that the parent report is negative), and (e) likelihood ratios estimates (the likelihood that a given parent report "test result" would be expected for a child with HKD compared with the likelihood of the same "test result" for a child without HKD)

4. Finally, amongst children who had a parent-only diagnosis, we examined the relative influence of the addition of the three different approaches (strict, medium, and loose) to conceptualising teacher ratings on the PPV for a DAWBA diagnosis of HKD.

\section{Results}

\section{Parent reports about symptoms at school}

Amongst the children identified by the skip rule, $18.2 \%$ of parents reported "a lot" of teacher complaints about inattention, $11.6 \%$ hyperactivity, and $9.8 \%$ impulsivity. Parent reports about symptoms at school were moderately correlated with teacher ratings (correlation coefficients of $0.31-0.36 ; P<0.001$ ) but, for inattention and impulsivity, slightly more strongly correlated with home ratings (correlation coefficients of $0.37-0.41 ; P<0.001$; Table 2). Across all combinations of measures, correlations were generally higher for boys than girls. There were no particular differences by child age.

The PPVs (Table 3) for parent reports of "a lot" of teacher complaints (the "strict" approach) about hyperactivity and inattention were less than $30 \%$ but just above $50 \%$ for impulsivity, reflecting the
Table 2 Correlations between parent and teacher ratings and parent reports about symptoms at school

\begin{tabular}{llll}
\hline & $\begin{array}{l}\text { Parent and } \\
\text { teacher ratings }\end{array}$ & $\begin{array}{l}\text { Teacher ratings and parent } \\
\text { reports about school }\end{array}$ & $\begin{array}{l}\text { Parent ratings and parent } \\
\text { reports about school }\end{array}$ \\
\hline $\begin{array}{l}\text { Inattention } \\
\text { Whole sample }\end{array}$ & $0.23(0.20,0.26)$ & $0.31(0.28,0.34)$ & \\
$\begin{array}{l}\text { Male/female } \\
\text { Younger/older }\end{array}$ & $0.24 / 0.21$ & $0.32 / 0.24$ & $0.40(0.37,0.43)$ \\
$\begin{array}{l}\text { Hyperactivity } \\
\text { Whole sample }\end{array}$ & $0.24 / 0.23$ & $0.34 / 0.28$ & $0.44 / 0.33$ \\
Male/female & $0.25(0.22,0.28)$ & $0.36(0.33,0.39)$ & $0.39 / 0.41$ \\
Younger/older & $0.26 / 0.18$ & $0.35 / 0.36$ & $0.37(0.34,0.40)$ \\
$\begin{array}{l}\text { Impulsivity } \\
\text { Whole sample }\end{array}$ & $0.25 / 0.23$ & $0.38 / 0.35$ & $0.39 / 0.28$ \\
Male/female & $0.21(0.18,0.24)$ & $0.33(0.30,0.36)$ & $0.39 / 0.35$ \\
Younger/older & $0.24 / 0.16$ & $0.34 / 0.27$ & $0.41(0.38,0.44)$ \\
\hline
\end{tabular}

$95 \% \mathrm{Cl}$ in brackets All $P<0.001$ 
Table 3 Predictive values of comparisons between teacher ratings and parent reports about ADHD symptoms at school

\begin{tabular}{|c|c|c|c|c|c|c|}
\hline \multirow[t]{2}{*}{$\begin{array}{l}\text { Teacher ratings } \\
\text { (number of symptoms) }\end{array}$} & \multicolumn{2}{|c|}{$\begin{array}{l}\text { Parent reports about } \\
\text { inattention symptoms } \\
\text { at school }\end{array}$} & \multicolumn{2}{|c|}{$\begin{array}{l}\text { Parent reports about } \\
\text { hyperactivity symptoms } \\
\text { at school }\end{array}$} & \multicolumn{2}{|c|}{$\begin{array}{l}\text { Parent reports about } \\
\text { impulsivity symptoms } \\
\text { at school }\end{array}$} \\
\hline & PPV (\%) & NPV (\%) & PPV (\%) & NPV (\%) & PPV (\%) & NPV (\%) \\
\hline Inattention $6+$ (strict) & 29 & 89 & - & - & - & - \\
\hline Inattention 3+ (middle) & 53 & 75 & - & - & - & - \\
\hline Hyperactivity 3+ (strict) & - & - & 27 & 94 & - & - \\
\hline Hyperactivity $2+$ (middle) & - & - & 41 & 90 & - & - \\
\hline Impulsivity $1+$ & - & - & - & - & 52 & 84 \\
\hline$\geq 6$ teacher symptoms (middle) & 46 & 82 & 57 & 81 & 57 & 80 \\
\hline$\geq 1$ teacher symptom (loose) & 75 & 54 & 82 & 53 & 82 & 52 \\
\hline
\end{tabular}

PPV positive predictive value, NPV negative predictive value

Table 4 Cut-off points for parent reports about symptoms at school in children with a parent-only diagnosis of hyperkinetic disorder

\begin{tabular}{|c|c|c|c|c|c|c|}
\hline $\begin{array}{l}\text { Parent reports of } \\
\text { symptoms at school (total) }\end{array}$ & PPV & NPV & Sensitivity & Specificity & $\begin{array}{l}\text { Likelihood } \\
\text { ratio +ve }\end{array}$ & $\begin{array}{l}\text { Likelihood } \\
\text { ratio -ve }\end{array}$ \\
\hline$\geq 1$ & 0.50 & 0.69 & 0.91 & 0.19 & 1.12 & 0.47 \\
\hline$\geq 2$ & 0.50 & 0.69 & 0.88 & 0.24 & 1.16 & 0.50 \\
\hline$\geq 3$ & 0.53 & 0.68 & 0.82 & 0.34 & 1.24 & 0.53 \\
\hline$\geq 4$ & 0.56 & 0.66 & 0.73 & 0.49 & 1.43 & 0.53 \\
\hline$\geq 5$ & 0.59 & 0.65 & 0.66 & 0.60 & 1.65 & 0.57 \\
\hline 6 & 0.62 & 0.53 & 0.59 & 0.69 & 1.90 & 0.59 \\
\hline
\end{tabular}

Likelihood ratio for +ve test result $=$ sensitivity $/(1-$ specificity $)$

Likelihood ratio for - ve test result $=(1-$ sensitivity $) /$ specificity

requirement for fewer symptoms to be endorsed. The NPVs were much higher. As the criteria for pervasiveness became looser, the PPVs increased and NPVs decreased.

\section{Predictive values for the diagnosis of HKD}

Amongst children passing the skip rule where information on symptoms at school had been provided by both teachers and parents, $5.4 \%(193 / 3,563)$ met criteria for a parent-only diagnosis in terms of having sufficient parent-rated symptoms and level of impairment. Amongst these children with a parentonly diagnosis, the prevalence of a DAWBA diagnosis of $\mathrm{HKD}$ was $46.9 \%$.

The PPV for a parent-only diagnosis meeting a DAWBA diagnosis of HKD was 47\% (95\% CI 40-54\%) and the NPV was $97 \%$ (95\% CI 97-98\%). We then examined whether this relatively poor PPV could be improved by taking account of parent reports about symptoms at school. The ROC curve indicated that a total score of 5 or above (on the 0-6 scale) provided the best prediction of a DAWBA diagnosis with a sensitivity of $66 \%$ and specificity of $60 \%$ (Table 4 ). When parents reported this high level of teacher complaints ("a lot" in two domains and "a little" in the third) in addition to sufficient parent-rated symptoms and impairment, the PPV for a DAWBA diagnosis was $59 \%$ (95\% CI 49-69\%). Increasing the cut-off to a total score of 6 increased the predictive power (PPV $=62 \%$; 95\% CI 51-73\%) and specificity (69\%) of these parental reports but at the expense of a reduction in sensitivity to $59 \%$. Amongst these children with a parent-only diagnosis (i.e. who had teacher data available), we then explored the effect of each one-point increase on the $0-6$ scale on the PPV for a DAWBA diagnosis. The PPV for each score between 0 and 5 was between 30 and $40 \%$. In contrast, the PPV for a score of 6 on the parent reports about symptoms at school scale was $62 \%$ (i.e. "a lot" of teacher complaints in all three domains). We also tested the effects of the addition of the three different approaches (strict, medium, and loose) to conceptualising teacher ratings on the PPV for a DAWBA diagnosis (Table 5). The PPVs steadily increased from

Table 5 Effects of teacher ratings on a DAWBA diagnosis in children with a parent-only diagnosis of hyperkinetic disorder

\begin{tabular}{ll}
\hline Teacher ratings & PPV \\
\hline$\geq 1$ symptom (loose) & 0.54 \\
$\geq 6$ symptoms (middle) & 0.72 \\
$\geq 3+2+1$ symptoms (middle) & 0.90 \\
$\geq 6+3+1$ (strict) & 0.94 \\
\hline
\end{tabular}


$54 \%$ (loose approach involving just one teacher-rated symptom) to $94 \%$ (strict approach requiring the teacher to endorse the presence of at least $6+3+1$ symptoms which is the ICD-10 domain-specific minimum) with increasing strictness of pervasiveness demands.

When compared by age group, the PPVs for the parent-only diagnosis meeting a DAWBA diagnosis were similar in younger $(44 \% ; 95 \%$ CI $35-53 \%)$ and older children (51\%; 95\% CI 39-64\%). However, there were gender differences with a PPV of $53 \%$ (95\% CI $45-61 \%$ ) in boys and 25\% (95\% CI $13-40 \%$ ) in girls. The ROC curve indicated that at a cut-off of 5 or above for parent reports of symptoms at school, the PPV for a corroborative rating of a DAWBA diagnosis was $64 \%(95 \%$ CI $53-74 \%)$ in boys and $36 \%$ (95\% CI $14-62 \%)$ in girls.

\section{Discussion}

In a large community sample, we found that although parent reports about HKD symptoms at school were associated with teacher ratings, they had limited utility at a diagnostic level. Our findings suggest that, amongst children whose parents report sufficient symptoms and impairment to potentially meet criteria for HKD as well as report high levels of symptoms at school, just over half are likely to meet diagnostic criteria for HKD. As the presence of HKD could be misdiagnosed in up to half of children passing the skip rule when relying solely on a parental account, this suggests that it is highly desirable to obtain teacher ratings.

Parent reports about symptoms at school were slightly more strongly correlated with home ratings than with teacher ratings. The strength of the correlation with teacher ratings was similar to that reported in referred children with ADHD [5]. However, the level of correlation with home ratings was much lower than expected which suggests the possibility that, in clinical samples, referral bias might influence parental accounts about school behaviour. The strength of the correlations was generally higher in boys than girls. In terms of their utility in predicting pervasiveness, we found that a negative parent report about symptoms at school was likely to be reliable, i.e. confirmed by the teacher. In contrast, when adopting a strict approach to pervasiveness, a positive report about symptoms at school is likely to be incorrect the majority of the time and teacher ratings are required. Although there may be a greater level of contact and exchange of information between teachers and parents in relation to younger children and boys, the magnitude of any differences was small. Teachers are likely to vary in the quantity and quality of their feedback to parents and this may be partly constrained by how much contact the parents choose to have. Also parents may misunderstand, project their own views onto teachers, or judge partly from their child's account of whether the teacher complains. As the criteria for pervasiveness were progressively relaxed with a requirement for only one teacher-rated symptom, the PPVs increased at the expense of the NPVs. Collectively, these findings provide some guidance about the possible utility of parent reports about symptoms at school if teacher ratings are unavailable.

At a diagnostic level, the PPV of a parent-only diagnosis for a DAWBA diagnosis of HKD was $47 \%$. If relying solely on a parental account of symptoms and impairment to make a diagnosis of HKD, this is only likely to be correct about half the time. As this is a community sub-sample, this is likely to be a minimum estimate-the PPV may be higher in clinically referred or other high-risk samples as predictive values are dependent on the prevalence of the disorder within the sample. However, the PPV is of relevance to children who potentially meet criteria for HKD in terms of sufficient parent-rated symptoms and impairment but do not have an available teacher report. These findings contrast with findings from referred samples which indicated that most children who met diagnostic criteria for ADHD based on parent report also met diagnostic criteria according to teacher report $[2,3,26]$. This discrepancy may reflect the higher prevalence rates of ADHD/HKD in referred samples, the possible influence of referral bias, our choice of "parent reports about school" measure or the use of different diagnostic criteria. In our sample, the addition of parent reports about symptoms at school modestly increased the PPV to 59\%; this is based on a particularly stringent cut-off as it reflects parent reports of "a lot" of symptoms (inattentiveness, overactivity, or impulsiveness) at school in at least two domains and "a little" in the third domain. The PPVs increased to $64 \%$ in boys, suggesting that the combination of parent-rated symptoms and impairment and parent reports about symptoms at school may have greater utility than in girls. This finding may also reflect the higher prevalence rates of HKD amongst boys.

\section{Methodological and research issues}

This study is based on a large, nationally representative, community sample and focuses on children whose parents, on questioning, report possible symptoms of HKD. The sample size allowed examination of gender and age effects. As the study is set in 
the UK, it is uncertain how well the findings generalise to other countries. The two datasets were based on identical methodology and provided very similar prevalence rates of disorder [11]. In the present study, the analyses were repeated using each dataset separately and provided a similar pattern of findings - any slight differences reflected the smaller sample size of the 2004 dataset. This sample of children identified by the skip rule is in keeping with practice recommendations for screening for $\mathrm{ADHD} / \mathrm{HKD}$ in a mental health assessment and where there is a need to carry out a more detailed evaluation [1].

The conceptualisation of HKD within ICD-10 and the fact that the clinical raters had access to the same data used in this study might have influenced the reported PPVs for HKD. However, these factors may only have a small effect on the findings as the analyses involving the parent-only diagnosis of HKD were restricted to children where teacher data were available. The clinician raters attributed more weight to the actual teacher data than to the parent reports about symptoms at school. When the effects of using the teacher data with increasing strictness of pervasiveness demands were examined, the findings highlighted that the lower PPVs obtained through using a single informant were not a function of any hierarchical or exclusionary criteria specified in the ICD-10 definition of HKD. In contrast, this substantial improvement in the PPV confirmed that teacher ratings can influence the receipt of a DAWBA diagnosis of HKD. This highlights the need for obtaining information from more than one source and that the addition of teacher reports provides additional useful information.

There are three main methodological limitations. First, for both illustrative purposes and to reflect the process of the initial parental presentation of problems to clinicians, our criteria for a parent-only diagnosis for HKD required that the parent endorsed a minimum number $(6+3+1)$ of symptoms in each domain. This requirement could be seen as too strict. To address this, our use of less stringent cut-offs to examine pervasiveness allowed for the detection of the presence of some symptoms. This is more relevant to usual clinical and research practice where reports are combined across informants so that a sufficient number of symptoms occur according to at least one informant, with each informant endorsing some symptoms. For ADHD, variations in the interpretation of whether symptoms are reported in either the home or school setting (an "either/or" approach) have been shown to influence prevalence rates [24].

Second, parent reports about school were restricted to teacher complaints about symptoms. As the diagnostic criteria for $\mathrm{HKD}$ ask for pervasive symptoms, teacher complaints about symptoms were investigated as predictors of teacher ratings of symptoms. To assess pervasiveness, ratings by the current teacher were treated as the "gold standard". Although parents were specifically asked whether the teachers have "complained" over the last 6 months, complaints are not the same as teacher reports about the child's behaviour to the parent. Hence, this is a strict test of whether parents are aware of symptoms of school. As the threshold at which a teacher might complain about these symptoms to the parent might vary considerably, the "no", "a little" and "a lot" coding of whether the teacher had complained provides some indication of the parental perception of the amount or frequency of teacher complaints. This parental perspective is important as this "secondhand" information might be reported if the parent presents concerns to the clinician. However, our findings may not fully generalise to other studies where different samples or measures are used. The presentation of our findings in Table 4 allows for comparisons with findings from future studies.

Third, this is a secondary analysis of a dataset from two cross-sectional surveys. As the surveys focused on current problems, parents were asked to report about "any" teacher complaints in the last 6 months rather than about "ever" complaints. Also it was not possible to assess teacher variation in ratings. In terms of research implications, a longitudinal study is required to fully account for the sequence over time of parentteacher communication about child behaviour. Future research should investigate the timing, frequency and persistence of teacher complaints across the academic year. This should also investigate parental accounts of impairment for the child at school (especially academic impairment) as pervasiveness of impairment is crucial in diagnostic decisions about ADHD.

\section{Clinical implications}

How useful are parent reports about symptoms at school in practice? To minimise the possibility of misdiagnosis, the pervasiveness of symptoms should play a key role in decisions about diagnosis and treatment. In a consultation with a parent concerned about HKD, a corroborative report from the teacher or other further enquiry is highly desirable even if the parental account is highly suggestive of HKD. However, arranging for the completion of teacher questionnaires carries considerable time implications for clinicians and schools. In routine practice, it is feasible to add these three questions involving parent reports about HKD symptoms at school to an assessment that already elicits parental accounts of symptoms and impairment. This set of questions is a partially useful adjunct to the assessment after a full 
evaluation has been carried out in the clinic but the teacher information is not available. However, the limited sensitivity and specificity values mean that we do not recommend these questions as a screening instrument at the population level. There are considerable risks of both false positives and negatives through sole reliance on parent reports. Teacher reports provide complementary and additional information and, as in clinical samples referred for assessments for $\mathrm{ADHD}$, reduce false positive rates [23]. Although parents are able to convey useful information about symptoms at school and these reports can substitute for teacher ratings when monitoring response to treatment [4], the present findings highlight the need for caution when assessing a child for a possible diagnosis of HKD if teacher reports are missing.

\section{Conclusions}

Although parent reports about their child's HKD symptoms at school partially correlate with and predict teacher ratings, they have limited utility in assisting with predictions at a diagnostic level. If it is not possible to obtain teacher information, clinicians and researchers should be aware that "second-hand" information about symptoms at school is second best. Our findings provide empirical support to clinical practice guidelines that it is highly desirable and worthwhile for clinicians to make the effort to obtain information from the teacher when making a diagnostic assessment for HKD.

\section{References}

1. American Academy of Child and Adolescent Psychiatry (2007) Practice parameter for the assessment and treatment of children and adolescents with attention-deficit/hyperactivity disorder. J Am Acad Child Adolesc Psychiatry 46:894-921

2. Biederman J, Keenan K, Faraone SV (1990) Parent-based diagnosis of attention deficit disorder predicts a diagnosis based on teacher report. J Am Acad Child Adolesc Psychiatry 29:698-701

3. Biederman J, Faraone SV, Milberger S, Doyle A (1993) Diagnoses of attentiondeficit hyperactivity disorder from parent reports predict diagnoses based on teacher reports. J Am Acad Child Adolesc Psychiatry 32:315-317

4. Biederman J, Faraone SV, Monuteaux MC, Grossbard JR (2004) How informative are parent reports of attentiondeficit/hyperactivity disorder symptoms for assessing outcome in clinical trials of long-acting treatments? A pooled analysis of parents' and teachers' reports. Pediatrics 113:1667-1671

5. de Nijs PF, Ferdinand RF, de Bruin EI, Dekker MC, van Duijn CM, Verhulst FC (2004) Attention-deficit/hyperactivity disorder (ADHD): parents' judgment about school, teachers' judgment about home. Eur Child Adolesc Psychiatry 13:315-320

6. Ford T, Goodman R (2005) The Ford Score; a simple measure for predicting the level of emotional and behavioural problems within mainstream school. Emotional Behavioural Difficulties 10:219-233
7. Ford T, Goodman R, Meltzer H (2003) The British Child and Adolescent Mental Health Survey 1999: the prevalence of DSM-IV disorders. J Am Acad Child Adolesc Psychiatry 42:1203-1211

8. Goodman R (1997) The strengths and difficulties questionnaire: a research note. J Child Psychol Psychiatry 38:581-586

9. Goodman R (2001) Psychometric properties of the strengths and difficulties questionnaire. J Am Acad Child Adolesc Psychiatry 40:1337-1345

10. Goodman R, Ford T, Richards H, Meltzer H, Gatward R (2000) The development and well-being assessment: description and initial validation of an integrated assessment of child and adolescent psychopathology. J Child Psychol Psychiatry 41:645-655

11. Green H, McGinnity A, Meltzer H, Ford TJ, Goodman R (2005) Mental health of children and young people, Great Britain 2004. Palgrave MacMillan, London

12. Meltzer H, Gatward R, Goodman R, Ford T (2000) Mental health of children and adolescents in Great Britain. The Stationery Office, London

13. Mitsis EM, McKay KE, Schulz KP, Newcorn JH, Halperin JM (2000) Parent-teacher concordance for DSM-IV attention-deficit/hyperactivity disorder in a clinic-referred sample. J Am Acad Child Adolesc Psychiatry 39:308-313

14. Rey JM, Sawyer MG (2003) Are psychostimulant drugs being used appropriately to treat child and adolescent disorders? Br J Psychiatry 182:284-286
15. Santosh PJ, Taylor E, Swanson J, Wigal T, Chuang S, Davies M et al (2005) Refining the diagnoses of inattention and overactivity syndromes: a reanalysis of the Multimodal Treatment study of attention deficit hyperactivity disorder (ADHD) based on ICD-10 criteria for hyperkinetic disorder. Clin Neurosci Res 5:307-314

16. Sawyer MG, Rey JM, Arney FM, Whitham JN, Clark JJ, Baghurst PA (2004) Use of health and school-based services in Australia by young people with attention-deficit/hyperactivity disorder. J Am Acad Child Adolesc Psychiatry 43:1355-1363

17. Sayal K, Taylor E (2005) Parent ratings of school behaviour in children at risk of attention deficit/hyperactivity disorder. Acta Psychiatr Scand 111:460-465

18. Sayal K, Taylor E, Beecham J (2003) Parental perception of problems and mental health service use for hyperactivity. J Am Acad Child Adolesc Psychiatry 42:1410-1414

19. Sayal K, Goodman R, Ford T (2006) Barriers to the identification of children with attention deficit/hyperactivity disorder. J Child Psychol Psychiatry 47:744-750

20. Scheffler RM, Hinshaw SP, Modrek S, Levine P (2007) The global market for ADHD medications. Health Aff 26:450457

21. Taylor E, Dopfner M, Sergeant J, Asherson P, Banaschewski T, Buitelaar J, Coghill D, Danckaerts M, Rothenberger A, Sonuga-Barke E, Steinhausen HC, Zuddas A (2004) European clinical guidelines for hyperkinetic disorder-first upgrade. Eur Child Adolesc Psychiatry 13(Suppl 1):7-30 
22. Tripp G, Luk SL, Schaughency EA, Singh R (1999) DSM-IV and ICD-10: a comparison of the correlates of ADHD and hyperkinetic disorder. J Am Acad Child Adolesc Psychiatry 38:156-164

23. Tripp G, Schaughency EA, Clarke B (2006) Parent and teacher rating scales in the evaluation of attention-deficit hyperactivity disorder: contribution to diagnosis and differential diagnosis in clinically referred children. J Dev Behav Pediatr 27:209-218
24. Wolraich ML, Lambert EW, Bickman L, Simmons T, Doffing MA, Worley KA (2004) Assessing the impact of parent and teacher agreement on diagnosing attention-deficit hyperactivity disorder. J Dev Behav Pediatr 25:41-47

25. World Health Organisation (1992) International Statistical Classification of Diseases and related health problems. WHO, Geneva Tenth Revision
26. Zeiner P (1997) Parent-reported symptoms of hyperactivity and attention deficits predict teacher-reported symptoms. Acta Paediatr 86:178-182 